\title{
Hash Value
}

National Cancer Institute

\section{Source}

National Cancer Institute. Hash Value. NCI Thesaurus. Code C68725.

An irreversible unique number generated from a string of text. The hash value is calculated so that it's unlikely, but not impossible, for some other text string to result in the same hash value, but the calculation guarantees that the same string will always produce the same hash value. 